\title{
DIMENSIONAL DEPENDENCE OF EXCHANGE INTERACTIONS AT HIGH MAGNETIC FIELDS
}

U. Zehnder, B. Kuhn-Heinrich, W. Ossau, A. WaAG, G. Landwehr Physikalisches Institut der Universität Würzburg Am Hubland, 97074 Würzburg, Germany

\section{H.H. Cheng and R.J. Nicholas}

Clarendon Laboratory, University of Oxford, Parks Road, Oxford OX1 3PU, UK

We studied the contribution of the breaking of antiferromagnetically coupled spin clusters to the total magnetization in thin (CdMn)Te layers as a function of the layer thickness by reflectivity spectroscopy in magnetic fields up to $45 \mathrm{~T}$. The experimental results show that the contribution of the breaking of antiferromagnetically coupled spin clusters is reduced by decreasing layer thickness.

PACS numbers: $78.55 . \mathrm{Et}, 71.35 . \mathrm{Ji}$

One of the characteristic features of diluted magnetic semiconductors like $(\mathrm{CdMn}) \mathrm{Te}$ is the giant Zeeman splitting of excitonic states. The Zeeman splittings are caused by two contributions. The first, important at low magnetic fields, is related to the alignment of single manganese spins and spin clusters by the magnetic field and is quantitatively well described by the mean spin model of Gaj [1] using a modified Brillouin function

$$
\left\langle\Delta E_{\sigma \pm}\right\rangle=0.5 x_{\mathrm{Mn}}\left(N_{0} \alpha-N_{0} \beta\right) S_{0} B_{5 / 2}\left[\frac{g \mu_{\mathrm{B}} B}{k\left(T_{\mathrm{eff}}+T\right)}\right],
$$

where $S_{0}$ denotes the effective spin, $T_{\text {eff }}$ - the effective temperature and $T$ - the lattice temperature. The values of the exchange parameters for $\mathrm{Cd}_{1-x} \mathrm{Mn}_{x} \mathrm{Te}$ are $N_{0} \alpha=220 \mathrm{meV}$ and $N_{0} \beta=-880 \mathrm{meV}$ [2].

The second contribution to the Zeeman splitting is caused by the breaking of antiferromagnetically coupled spin clusters in high magnetic fields. A description of both contributions is given by an empirical model of Nicholas et al. [3] combining two modified Brillouin functions 


$$
\begin{aligned}
& \left\langle\Delta E_{\sigma \pm}\right\rangle=0.5 x_{\mathrm{Mn}}\left(N_{0} \alpha-N_{0} \beta\right) \\
& \quad \times\left[S_{0}+\left(5 / 2-S_{0}\right) B_{5 / 2}\left(\frac{g \mu_{\mathrm{B}} B}{k T_{2}}\right)\right] B_{5 / 2}\left(\frac{g \mu_{\mathrm{B}} B}{k T_{1}}\right),
\end{aligned}
$$

where $S_{0}$ denotes the effective spin and $T_{1}, T_{2}$ are effective temperatures. The revised parameter $S_{0}$ more accurately reflects the mean spin at $B=0 \mathrm{~T}$. It turns out that the temperature $T_{1}$ corresponds to the lattice temperature [3], whereas the antiferromagnetic temperature $T_{2}$ is characteristic of the second contribution to the total magnetization.

The relative influence of both contributions to the Zeeman splitting is illustrated in Fig. 1, where we plot the experimental data for the lower branch of the Zeeman splitting of the heavy hole exciton $\left(\Delta E_{\sigma+}\right)$ of a $\mathrm{Cd}_{0.82} \mathrm{Mn}_{0.18}$ Te layer at $4.2 \mathrm{~K}$. The data have been obtained by reflectivity spectroscopy in pulsed magnetic fields up to $45 \mathrm{~T}$ [4]. Descriptions after the models of Nicholas and Gaj are shown by lines. The model of Gaj gives a satisfactory description for magnetic fields up to $10 \mathrm{~T}$, while the second part becomes more important for higher magnetic fields.

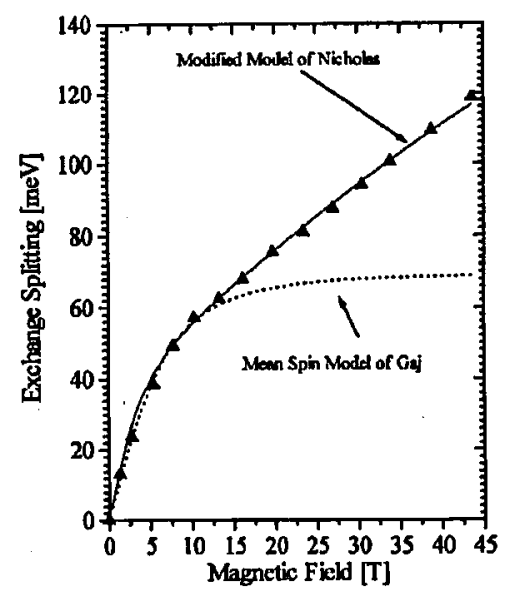

Fig. 1. Lower branch of exchange splitting of the heavy hole exciton of a $\mathrm{Cd}_{0.82} \mathrm{Mn}_{0.18} \mathrm{Te}$ layer. The data are taken by reflectivity spectroscopy in pulsed magnetic fields at $4.2 \mathrm{~K}$. The solid line reflects the best fit of the data by the Nicholas model and dashed line by the model of Gaj.

As the effective temperature $T_{2}$ describes the breaking of antiferromagnetically coupled spin clusters it is interesting to study the influence of the reduction of the layer thickness on the effective temperature $T_{2}$. For this purpose we have investigated the Zeeman splittings of $\mathrm{Cd}_{1-x} \mathrm{Mn}_{x} \mathrm{Te} / \mathrm{Cd}_{1-y} \mathrm{Mg}_{y} \mathrm{Te}$ quantum wells. The well widths vary from $12 \AA$ to $300 \AA$ and the manganese contents are $x=0.10$ and $x=0.18$. The magnesium concentrations are $y=0.36$ and $y=0.45$, respectively, to get a comparable confinement within the investigated structures. 
We have fitted the experimental data by Eq. (2) and, for low fields $(B<$ $10 \mathrm{~T}$ ) additionally by Eq. (1), after eliminating the well width dependent influences of the diamagnetic shift and the overlap of the wave function with the semimagnetic layer.

The results are shown in Fig. 2. For the low field case we observe a decrease in the effective temperature $T_{\text {eff }}$ with decreasing well width. The decrease in $T_{\text {eff }}$ is more pronounced for higher manganese contents (Fig. 2b), which is already discussed by Ossau et al. [5]. The antiferromagnetic temperature $T_{2}$ also decreases with decreasing well width and manganese content, in a similar way to the effective temperature $T_{\text {eff }}$ (Fig. 2a).

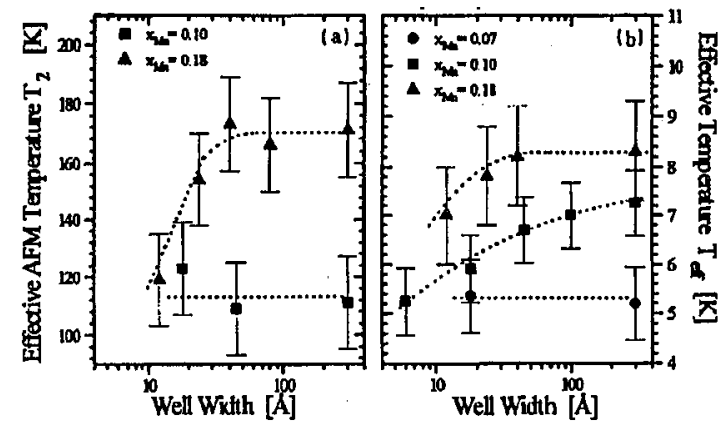

Fig. 2. (a) Effective antiferromagnetic temperature $T_{2}$ for $\mathrm{Cd}_{1-x} \mathrm{Mn}_{x} \mathrm{Te} / \mathrm{Cd}_{1-y} \mathrm{Mg}_{y} \mathrm{Te}$ heterostructures fitted with the Nicholas model. The manganese contents are $x=0.10$ and $x=0.18$. (b) Effective temperature $T_{\text {eff }}$ for the same structures as in (a) fitted with the Gaj model. The data are taken with photoluminescence excitation spectroscopy in magnetic fields up to $8 \mathrm{~T}$.

The reduction of the layer thickness increases the ratio between the numbers of spins located at the interface and in the volume of the semimagnetic material resulting in smaller antiferromagnetically coupled spin clusters compared to bulk material [6]. This affects both contributions to the Zeeman splitting. On the one hand, the manganese spins in thin layers are more easily aligned than in bulk material, resulting in a more paramagnetic behaviour of the structure. Enhanced paramagnetic behaviour is equivalent to a reduction of $T_{\text {eff }}[6]$. On the other hand, caused by the reduction of the number of spin clusters, the breaking of the antiferromagnetically coupled spin clusters in high magnetic fields contributes less to the total magnetization which is reflected by the behaviour of $T_{2}$. Therefore, a reduction of the layer thickness changes the ratio between the two contributions to the magnetization.

In conclusion, the breaking of antiferromagnetically coupled spin clusters gives a significant contribution to the total magnetization of semimagnetic layers in high magnetic fields. For the first time we have demonstrated experimentally that this contribution is drastically affected by the reduction of the layer thickness of the magnetic layer. 
The authors wish to thank the Deutsche Forschungsgemeinschaft for the financial support through Sonderforschungsbereich 410 as well as by Graduiertenkolleg.

\section{References}

[1] J.A. Gaj, R. Planel, G. Fishman, Solid State Commun. 29, 435 (1979).

[2] J.K. Furdyna, J. Appl. Phys. 64, R29 (1988).

[3] R.J. Nicholas, M.J. Lawless, H.H. Cheng, D.E. Ashenford, B. Lunn, Semicond. Sci. Technol. 10, 791 (1995).

[4] H. Jones, R.G. Jenkins, M. Van Cleemput, R.J. Nicholas, W.J. Siertsma, M. van der Burgt, Physica B 201, 546 (1994).

[5] W. Ossau, B. Kuhn-Heinrich, A. Waag, G. Landwehr, J. Cryst. Growth 159, 1046 (1996).

[6] W. Ossau, B. Kuhn-Heinrich, Physica B 184, 422 (1992). 